\title{
Data Analysis of Pile-free bike-sharing from Market Status to Operation Management Mode
}

\author{
Jingyu Liang, Yiqing Zhu, Peixue Lin \\ Beijing Jiaotong University, Beijing Haidian ,100044
}

\begin{abstract}
Shared travel plays a more and more active role in the emergence of urban traffic, but there is no systematic and perfect combing and analysis on the study of bike-sharing without piles, especially on its operation and management mode. This paper will conduct a comprehensive data analysis on the research of bike-sharing without piles from the individual micro level and the enterprise government level, and explore the research focus and possible subject areas of future research.
\end{abstract}

\section{1 introduction}

With the rapid development of bike-sharing market, bikesharing has become a research hotspot in recent years. At present, there have been rich achievements in the research of bike-sharing. The purpose of this study is to make a comprehensive review of the research on non-pile bikesharing from the individual micro level and the corporate government level.

This research uses three main steps to explore the research focus and possible subject areas of future research. At first, the literature about bike-sharing is collected from the Web of Science by using keywords such as dockless shared bicycle, dockless bike sharing, freefloating shared bicycles and freefloating bike sharing. Secondly, using literature analysis, this paper discusses the statistical characteristics of the collected literature, including: general characteristics, research areas and institutions, and research priorities. Finally, we put forward the main contents of the current literature and the future research direction from the bibliometric and thematic discussion.

\section{2 general characteristics of literature}

We use Citespace to analyze bike-sharing literature, which is an information visualization software developed by Chen Chao Mei team of Dalian University of Technology. By processing related literature, we can detect the hot topics and their evolution of a certain discipline. Our data comes from the Web of Science database, which can provide comprehensive and standardized information from literature.

On November 8th, 2020, we searched by using keywords such as dockless shared bicycle, dockless bike sharing, freefloating shared bicycles, freefloating bike sharing, etc. Since the bike-sharing without piles rose rapidly depending on the Internet in 2015, we focused on limiting the scope from 2015 to now, and finally identified 131 literatures, including 128 articles and 3 reviews.

\section{1 general characteristics}

Figure 1 describes the release time of our collected literature. The bike-sharing without piles appeared on a large scale in China in 2015. The research on it just started in 2016 and 2017, and reached 11 articles in 2018. However, the literature on bike-sharing increased rapidly in 2019 and 2020, and it is expected that the research literature on bike-sharing without piles will continue to rise in 2020 and beyond. 


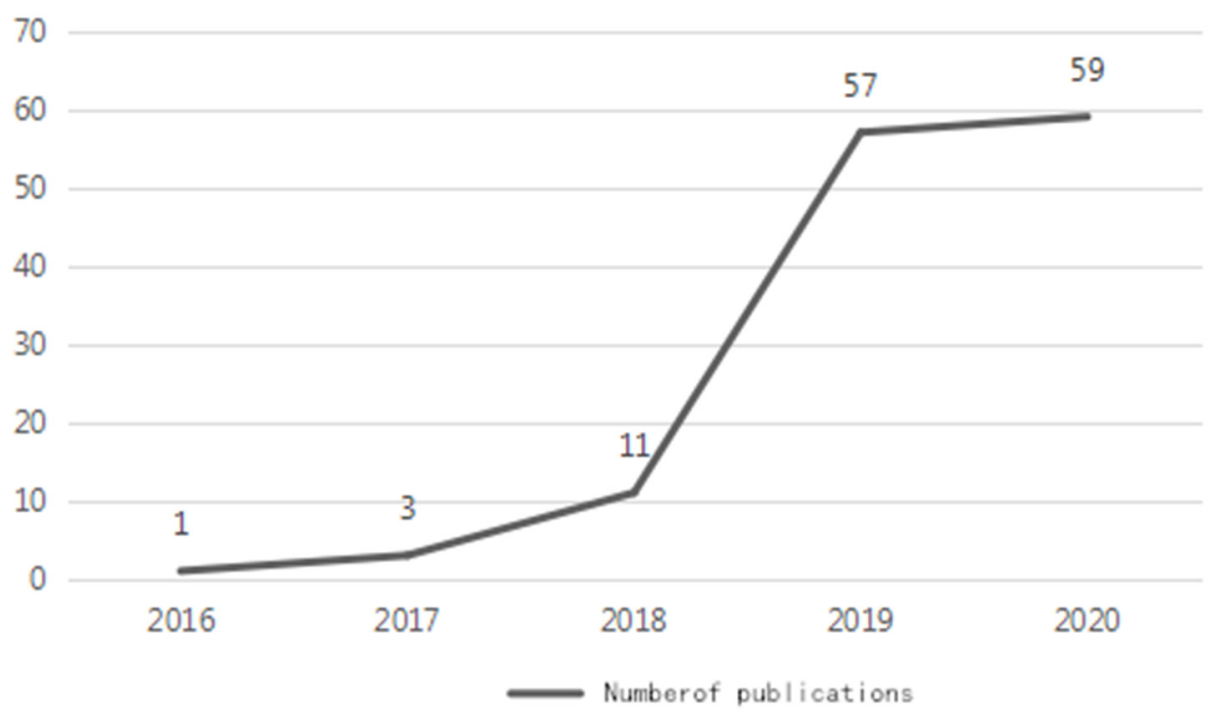

Figure 1 Number of documents published in each year

\section{2 research areas and institutions}

Table 1 shows that although the Netherlands started the research on bike-sharing without piles in 2016, the research on bike-sharing was more than that in China, the United States and Britain in 2017. Due to the successful spread of the non-pile bike-sharing business model in China, China leads other countries with 84 articles published.

In terms of research institutions, the top two universities are also from China-Southeast University and Tongji University, and the top six universities from the Netherlands and Australia also appear.

Table 1 Distribution of Literature Research Countries

\begin{tabular}{cccc}
\hline \hline Number of publications & centrality & Year of occurrence & country \\
84 & 1.30 & 2017 & China \\
25 & 0.22 & 2017 & United States of America \\
15 & 0.48 & 2017 & Britain \\
11 & 0.03 & 2016 & the Netherlands \\
8 & 0.18 & 2018 & Australia \\
7 & 0.03 & 2019 & Canada \\
5 & 0.00 & 2019 & Germany \\
5 & 0.03 & 2019 & Sweden \\
5 & 0.03 & 2018 & Singapore \\
\hline
\end{tabular}

Table 2 Distribution of Literature Research Institutions

\begin{tabular}{lll}
\hline \hline Number of publications & Year of occurrence & research institute \\
19 & 2018 & Southeast China University \\
17 & 2017 & Tongji University \\
5 & 2016 & Utrecht University \\
4 & 2019 & Shanghai Jiao Tong University \\
4 & 2018 & Beijing Jiaotong University \\
4 & 2018 & Monash university \\
\hline \hline
\end{tabular}




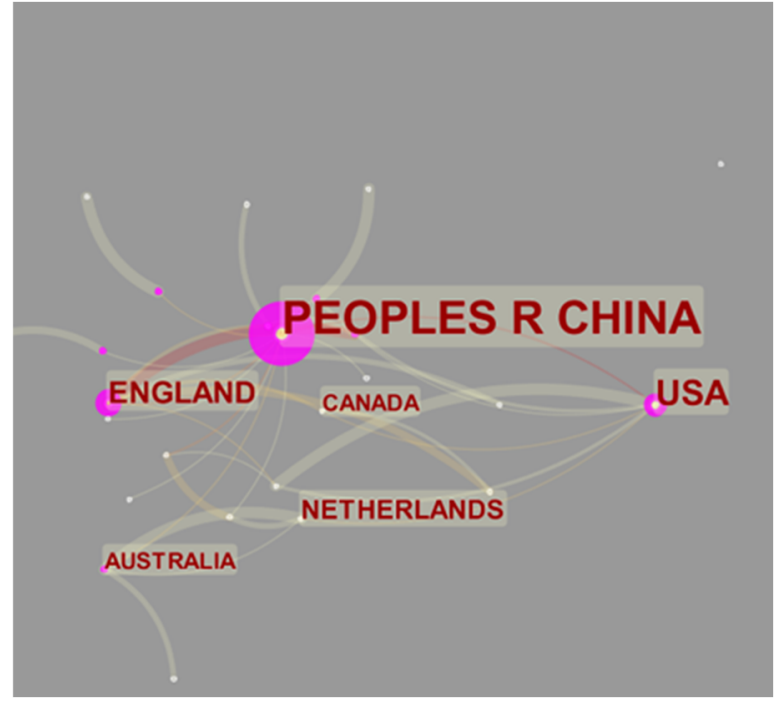

Figure 2 Distribution map of literature research countries

\section{3 research focus}

Key words are widely used to grasp the essence of articles, which can reveal the development trend of research hotspots. We can clearly and intuitively see the research trend in this field by analyzing the keywords in the literature with Citespace and drawing according to the first appearance time. At the time of the rise of bike-sharing, people were concerned about the influence and function of using bike-sharing on life and travel. From 2017 to 2019, on the one hand, people began to pay attention to users' behaviors and choices for using bike-sharing and the determinants affecting people's behavior; On the one hand, people began to pay attention to the algorithm generated by bicycle evolution, and how to optimize the allocation of resources invested by operators. Recently, people began to regard bike-sharing as a part of dynamic transportation, and made in-depth research and exploration on its built environment.

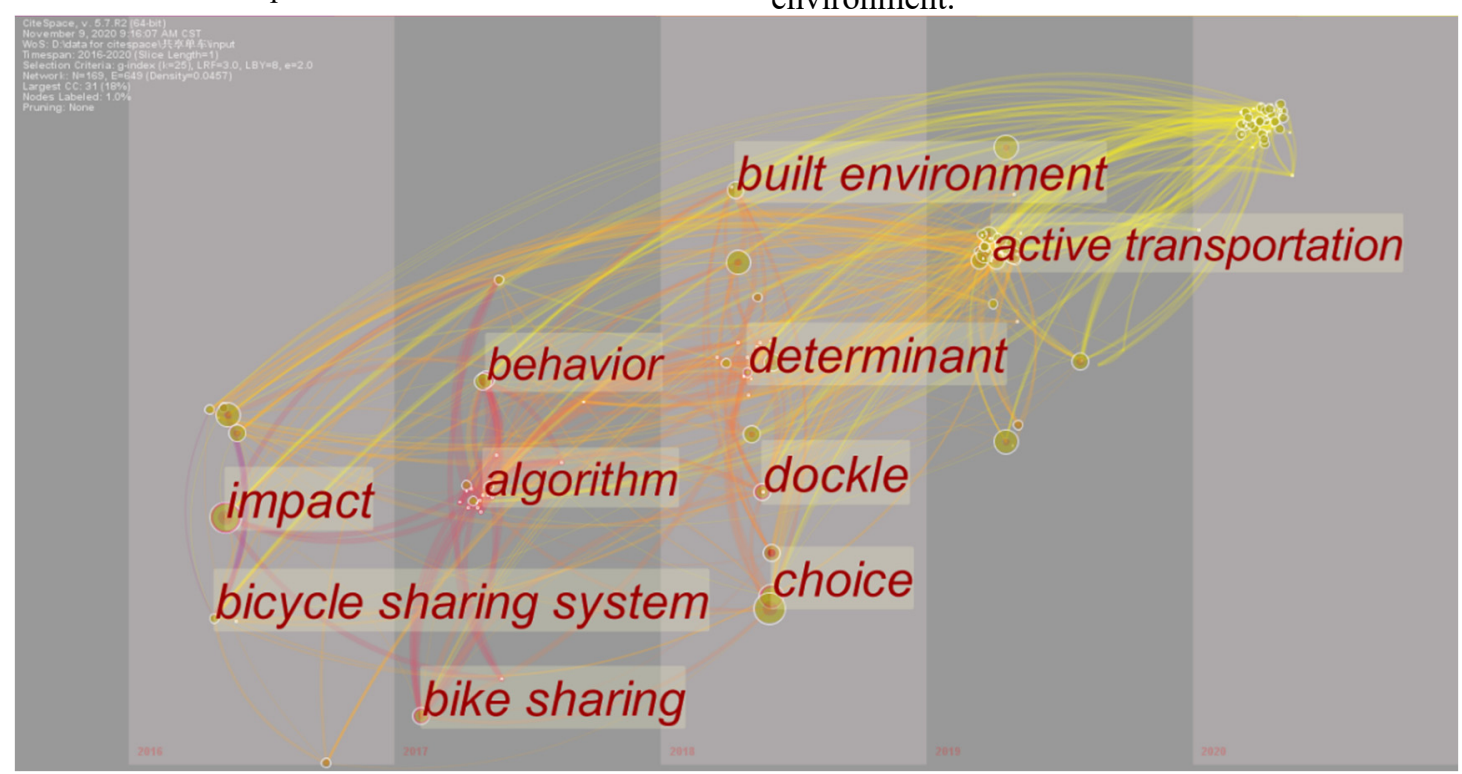

Figure 3 Literature keyword map

\section{3 literature research and analysis}

\subsection{Use of bike-sharing}

3.1.1 Travel behavior The research on the travel behavior of users in bike-sharing mainly includes three aspects, namely, the characteristics of users in bikesharing $[1][2][3]$, the competition and complementary relationship with other modes of transportation from the commuter's point of view ${ }^{[4][5][6][7]}$, and the spatial and temporal distribution characteristics used in bike-sharing [8][9].

1) user characteristics User characteristics include gender, age, education level, income level, residence and other characteristics. From the perspective of gender, male users are more common than female users ${ }^{[10]}$, which may be because it is more difficult for women to obtain and use bike-sharing than men. In terms of age, most of the users in bike-sharing are 35-year-old young people, and the old people use them less, which may be due to the advantages of low cost, convenience and rapidity that bike-sharing is more likely to attract young people. From the perspective of education level, people with higher education level are more willing to use bike-sharing, which may be because users with higher education level are more likely to accept new technologies. From the perspective of income level, the income level of most users in bike-sharing is higher than the social average level, which may be because highincome people are more willing to pay for the use of bikesharing. From the point of view of residence, there are more users in bike-sharing in communities with larger population, while there are fewer users in bike-sharing in communities with more ethnic diversity ${ }^{[11]}$. In addition, some people discussed the relationship between the use of bike-sharing and the means of transportation owned by families, and found that most users in bike-sharing do not have cars or electric bicycles.

2) From the perspective of commuters, the competition and complementary relationship with other modes of 
transportation Influenced by travel time, travel purpose, travel mode and user characteristics, there is a competitive and complementary relationship between bike-sharing and other modes of transportation. In the competitive relationship between bike-sharing and other modes of transportation, bike-sharing has a competitive relationship with buses, Uber and bike-sharing with piles, especially the impact on the use of bike-sharing with piles. In the competitive relationship with buses, the use of bikesharing leads to a decrease in the number of shared routes and bus passengers on weekends. In the competitive relationship with Uber, temporary users take bike-sharing and Uber as alternative products $\left.{ }^{[12}\right]$. In the competitive relationship with the piled bike-sharing, the launch and use of the piling-free bike-sharing system resulted in a significant decrease in the weekly average usage of each LCH docking station, and the short-distance (1-0) km trip of LCH was replaced by the short-distance bicycle (1-0 $\mathrm{km}$ ), while the trip of midway $\mathrm{km}$ also decreased, especially in the peak hours of commuting on weekdays and leisure on weekends ${ }^{[13]}$.

In the complementary relationship between bikesharing and other modes of transportation, bike-sharing has a complementary relationship with bus, subway, Uber, walking and other modes of transportation, and the proportion of bicycles combined with public transportation is relatively high, which improves the experience of taking public transportation ${ }^{[14]}$. In the complementary relationship with buses, the use of bikesharing improves the occupancy rate of buses on weekdays. In the complementary relationship with the subway, the utilization rate of bike-sharing is high on the route starting or ending with the subway/railway connection ${ }^{[15]}$. In the complementary relationship with Uber, banning Uber leads to a significant decline in the utilization rate of users who frequently use bike-sharing, especially on weekdays, which indicates that there is a significant complementary relationship between bikesharing and Uber. In the complementary relationship with walking, most users habitually use BSS only or combine it with walking [16]. In addition, some scholars have pointed out that it is necessary to improve the use of intermodal transportation between bike-sharing and other public or private means of transportation.

3)Spatio-temporal distribution In terms of time, bikesharing is mainly used in the peak hours of commuting on weekdays and afternoons, while the peak hours of use in bike-sharing still exist on weekends, but not as obvious as on weekdays. In terms of space, the demand for use in bike-sharing on weekdays is relatively concentrated, while the demand for use in bike-sharing on weekends is relatively scattered. In addition, the use of bike-sharing is mostly concentrated in densely populated communities. In areas with relatively dense public transport networks, the higher the utilization rate in bike-sharing. Activities related to daily life, such as catering and transportation, are widely distributed in bike-sharing, while activities related to work and shopping are concentrated in specific places $^{[17]}$.

\subsection{2 parking behavior The research on parking}

behavior of users in bike-sharing mainly includes three aspects, namely, the causes of disorderly parking [18][19][20], parking intention and influencing factors [21][22], and solving the problem of disorderly parking [23][24][25].

1) the reason of disorderly parking By constructing six factors and 32 standards, this paper studies the reasons of disorderly parking of shared bicycles. It is found that the three main objects of disorderly parking of shared bicycles are users, bicycle sharing companies and the government. From the user's point of view, due to the strong positive social interaction between bicycle owners, the collective behavior of bicycle parking is quite different among each group $^{[26]}$, and the game contradiction between cyclists and management departments is because both parties, as rational people, want to minimize their transaction costs and maximize their utility for the unclear property rights in bike-sharing parking area; From the company's point of view, the higher the management cost, the easier it is for the management department to choose not to manage; Generally speaking, there are three main reasons: lack of self-discipline of users, inadequate supervision and management of users by bike-sharing companies, government and society, and inadequate management and supervision of enterprises by the government.

2) Parking intention and influencing factors Parking intention and influencing factors mainly include social norms, subjective norms, consequence perception and system perception, education level and government policies.

From the perspective of social norms, social norms, reciprocity, communication responsibility and institutional environment play an important role in regulating and influencing parking behavior in bikesharing, and may be more important than monetary incentives. Reciprocity and responsibility play an important role in regulating and promoting parking behavior in bike-sharing. From the perspective of subjective norm, consequence perception and system perception, perceptual behavior control, consequence perception, behavior attitude, system perception and subjective norm can positively influence behavior intention, but their forces weaken in turn; From the perspective of education level, income level and education level have a significant impact on the use of bike-sharing. Residents with higher income level and education level are more willing to use bike-sharing, and bike-sharing users who have a clear understanding of orderly parking standards are more willing to regulate parking; From the perspective of government policies, the government can formulate relevant policies to guide people to regulate parking, including establishing orderly parking standards, posting slogans, delaying troubleshooting and lack of policies, which have a negative impact on people's willingness to use.

3) Solve the problem of random parking and misplacing There are three main ways to solve the problem: to discuss service design, to study technical means and to discuss policy compliance.

In the aspect of service design, we can design a lowcarbon bicycle parking service system through service design to solve the bicycle sharing problem of disorderly 
parking. PCN analysis method is based on the improvement of service blueprint method, which describes the problem of service network, improves social satisfaction and corporate image, saves operating costs for the company, and expands the possibility of other enterprises to improve their competitiveness; In terms of technical means, we should plan a sufficient number of electronic fences at suitable locations, and the method of electronic fences is suitable for all types of bicycles; It is also possible to establish a two-level FFBS monitoring and early warning index, which takes into account the realtime parking quantity and land use capacity of FFBS. The parking management strategy of FFBS aiming at different early warning indexes is put forward. This is very important for FFBS and the sustainable development of cities. In terms of policy compliance, both monetary rewards and fines will encourage people to park their shared bicycles in unsaturated places near their destinations. With the increase of incentive intensity, monetary rewards can encourage users of shared bicycles to move their bicycles away more effectively than fines, especially when they need to walk for more than 10 minutes. In addition, some factors used to characterize individual heterogeneity, such as gender, education level and search time in bike-sharing, also have a significant impact on the policy of abiding by the bicycle parking guide. These findings can help policy makers formulate appropriate measures in the form of incentives to reduce illegal parking of shared bicycle users.

\section{2 bike-sharing operation mode}

\subsubsection{Enterprises}

Careful planning of traffic configuration, system design and business model selection are the keys to a sustainable and successful bike-sharing system. The rapid growth of bus projects without piles is mainly driven by the supply of operators, rather than triggered by user demand or government policy. Geographical diffusion itself is a business strategy for entrepreneurs to manage competition [27].

At present, the main problems faced by operators in bike-sharing focus on inadequate system scale, site density, geographical coverage, ease of use, price structure, users' vandalism and their own financial sustainability [28][29][30]. Especially in parking, both parties, as rational people, want to minimize their transaction costs and maximize their utility for the parking area in bike-sharing, which has unclear property rights. The resistance of users and project participants to changes also hindered the further advancement of bike-sharing ${ }^{[31]}$.

In view of the current problems, many scholars put forward relevant suggestions: enterprises should strengthen the supervision and management of users, formulate clear parking standards or regulations in bikesharing, provide users with reasonable and sufficient parking space, and set up some guiding signs to facilitate users to find parking spaces. Investment in operation and maintenance should be increased, and sufficient operation and maintenance personnel should be provided. At the same time, the boundaries between the whole parking space and individual parking units should be clearly defined to help users park bike-sharing in an orderly manner. Electronic fences are introduced to solve the problem of random parking and misplacing by technical means.

It is suggested that the use of personal credit cards (allowing members in bike-sharing to pay "personal points" instead of money without returning public bicycles during the free use time) and universal cards (the number of rechargeable smart cards that can cover a series of payment and travel by integrating the bike-sharing system into other urban transportation systems) can significantly improve the daily use and turnover rate of bike-sharing. ${ }^{[32]}$ Based on the service blueprint, the PCN analysis method is improved, and a low-carbon bicycle parking service system is designed, which saves the operating cost for the company and improves the possibility of enterprise competitiveness. The generalized extremum counting model is used to analyze the demand pattern in urban bikesharing system. It enables bike-sharing customers to plan their trips better according to the expected bicycle parking conditions at the start and end of their trips, and also enables bike-sharing operators to better predict future demand, optimize their trips and rebalance their plans. A two-level FFBS monitoring and early warning index is established, which considers the real-time parking quantity and land use capacity. Put forward parking management strategies for different early warning indicators. It is pointed out that when the number of designed parking spots increases, the cost of each user will decrease correspondingly, and with the increase of the total cost of the system, the optimal distribution of parking spots is found based on Pareto frontier results.

3.2.2 The Government On the government side, some scholars believe that the government's inadequate management and supervision of enterprises is one of the main reasons for the phenomenon of disorderly stop and release in bike-sharing. Some scholars have also made suggestions to the government. For example, the government can formulate relevant policies to guide people to regulate parking, including establishing standards for orderly parking and posting slogans; Policymakers can formulate appropriate measures in the form of incentives to reduce illegal parking of shared bicycle users. At the same time, some scholars encourage policy makers to consider the value and meaning of "shared" platform more carefully ${ }^{[33]}$.

3.2.3 Tripartite coordination In order to solve the problem of random parking and littering in bike-sharing, many scholars believe that we should not rely solely on the strength of government, market or users, but establish a tripartite cooperation model of "government-enterpriseusers" ${ }^{[34][35]}$ or "government-market-society" to promote the sustainable development of bike-sharing. In the tripartite cooperation mode of "government-enterpriseuser", the government can carry out propaganda and guidance and spiritual encouragement; Enterprises mainly carry out material incentives; Users can improve the 
parking awareness of bike-sharing. The government can also strengthen supervision, enterprises should improve related infrastructure, and users should have a higher sense of responsibility. It is also pointed out that this collaborative governance should be a hierarchical and interrelated model ${ }^{[36]}$. In the tripartite cooperation mode of "government-market-society", the government should intervene properly, and the society should assist the government in management, thus forming a benign interaction among government, society and market [37]. Some people also discussed the main obstacles and cooperation modes of collaborative governance among government, society and market ${ }^{[38]}$. In addition, some people have studied the service mode of tripartite cooperation, and think that a new public service mode with market leading, government guiding and citizen participation should be established [39]. In addition, although some scholars have not explicitly pointed out the concept of "tripartite cooperation", they already have a concept similar to "tripartite cooperation" in measures. Some scholars think that users should strengthen their own behavior constraints, and companies, governments and society should strengthen supervision. From the perspective of sustainable development in bike-sharing in the future, some scholars believe that users should constantly improve their demand for using bike-sharing, the government should increase support, and enterprises should increase supply.

In addition to the governance model of tripartite cooperation, some scholars have refined the subject of tripartite cooperation into universities, urban management, street offices and other subjects, and discussed four levels of countermeasures ${ }^{[40]}$.

\section{4 summary}

Shared travel has a significant and positive impact on people's daily life, but there is no systematic review of the study of bike-sharing without piles. In this paper, a comprehensive review of the research on pile-free bikesharing is made from the individual micro level and the enterprise government level. We mainly use three main steps to describe and analyze the 131 documents on pilefree bike-sharing collected on the Web of Science from travel behavior to operation mode, and explore the research focus and possible subject areas of future research. A notable limitation of our research is that we rely on one database-Web of Science to apply bibliometrics. In future research, we can consider using additional literature databases and reviewing these articles more logically and orderly.

\section{References}

1. Chen, Mengwei, et al. "A Comparison of Users' Characteristics between Station-Based Bikesharing System and Free-Floating Bikesharing System: Case Study in Hangzhou, China." Transportation, vol. 47, no. 2, 2020, pp. 689-704.

2. Gu, Tianqi, et al. "To Be or Not to Be Dockless: Empirical Analysis of Dockless Bikeshare
Development in China." Transportation Research Part A-Policy and Practice, vol. 119, 2019, pp. 122-147.

3. Li, Xuefeng, et al. "Free-Floating Bike Sharing in Jiangsu: Users' Behaviors and Influencing Factors." Energies, vol. 11, no. 7, 2018, p. 1664.

4. Ma, Xiaolei, et al. "Impacts of Free-Floating Bikesharing System on Public Transit Ridership." Transportation Research Part D-Transport and Environment, vol. 76, 2019, pp. 100-110.

5. Bakó, Barna, et al. "Does Uber Affect BicycleSharing Usage? Evidence from a Natural Experiment in Budapest." Transportation Research Part A-Policy and Practice, vol. 133, 2020, pp. 290-302.

6. Kong, Hui, et al. "Deciphering the Relationship between Bikesharing and Public Transit: Modal Substitution, Integration, and Complementation." Transportation Research Part D-Transport and Environment, vol. 85, 2020, p. 102392.

7. Molinillo, Sebastian, et al. "User Characteristics Influencing Use of a Bicycle-Sharing System Integrated into an Intermodal Transport Network in Spain.” International Journal of Sustainable Transportation, vol. 14, no. 7, 2020, pp. 513-524.

8. Ma, Xinwei, et al. "A Comparison in Travel Patterns and Determinants of User Demand between Docked and Dockless Bike-Sharing Systems Using MultiSourced Data." Transportation Research Part APolicy and Practice, vol. 139, 2020, pp. 148-173.

9. Almannaa, Mohammed H., et al. "Dynamic Linear Models to Predict Bike Availability in a Bike Sharing System." International Journal of Sustainable Transportation, vol. 14, no. 3, 2020, pp. 232-242.

10. Ricci, Miriam. "Bike Sharing: A Review of Evidence on Impacts and Processes of Implementation and Operation." Research in Transportation Business and Management, vol. 15, 2015, pp. 28-38.

11. Brown, Anne E. "Who and Where Rideshares? Rideshare Travel and Use in Los Angeles." Transportation Research Part A-Policy and Practice, vol. 136, 2020, pp. 120-134.

12. Bakó, Barna, et al. "Does Uber Affect BicycleSharing Usage? Evidence from a Natural Experiment in Budapest." Transportation Research Part A-Policy and Practice, vol. 133, 2020, pp. 290-302.

13. Li, Haojie, et al. "Effects of Dockless Bike-Sharing Systems on the Usage of the London Cycle Hire." Transportation Research Part A-Policy and Practice, vol. 130, 2019, pp. 398-411.

14. Kong, Hui, et al. "Deciphering the Relationship between Bikesharing and Public Transit: Modal Substitution, Integration, and Complementation." Transportation Research Part D-Transport and Environment, vol. 85, 2020, p. 102392.

15. Böcker, Lars, et al. "Bike Sharing Use in Conjunction to Public Transport: Exploring Spatiotemporal, Age and Gender Dimensions in Oslo, Norway." Transportation Research Part A-Policy and Practice, vol. 138, 2020, pp. 389-401. 
16. Molinillo, Sebastian, et al. "User Characteristics Influencing Use of a Bicycle-Sharing System Integrated into an Intermodal Transport Network in Spain." International Journal of Sustainable Transportation, vol. 14, no. 7, 2020, pp. 513-524.

17. Chen, Zhiwei, et al. "Exploring the Equity Performance of Bike-Sharing Systems with Disaggregated Data: A Story of Southern Tampa." Transportation Research Part A-Policy and Practice, vol. 130, 2019, pp. 529-545.

18. Jiang, Qianling, et al. "Why Shared Bikes of FreeFloating Systems Were Parked Out of Order? A Preliminary Study Based on Factor Analysis." Sustainability, vol. 11, no. 12, 2019, p. 3287.

19. Fukuda, Daisuke, and Shigeru Morichi. "Incorporating Aggregate Behavior in an Individual's Discrete Choice: An Application to Analyzing Illegal Bicycle Parking Behavior." Transportation Research Part A-Policy and Practice, vol. 41, no. 4, 2007, pp. 313-325.

20. Dong Zhaoying, Wang Qiaoyun. Mixed strategy analysis and suggestions on disorderly parking in bike-sharing — Taking Ningbo North Higher Education Park as an example [J]. Chinese Market, 2019(22):24-26.

21. Yang Liuhua, Zhu Dajian. Analysis of influencing factors of bike-sharing's intention to regulate parking behavior under the framework of extended planned behavior theory $[\mathrm{J}]$. china population resources and environment, 2018,28(04):125-133.

22. Wang, Yacan, et al. "Factors Affecting Orderly Parking of Dockless Shared Bicycles: An Exploratory Study." International Journal of Logistics-Research and Applications, 2020, pp. 1-23.

23. Zhang, Yongping, et al. "Electric Fence Planning for Dockless Bike-Sharing Services.” Journal of Cleaner Production, vol. 206, 2019, pp. 383-393.

24. Xu, Dandan, et al. "Study on Clustering of FreeFloating Bike-Sharing Parking Time Series in Beijing Subway Stations." Sustainability, vol. 11, no. 19, 2019, p. 5439.

25. Gao, Liangpeng, et al. "Incentive Measures to Avoid the Illegal Parking of Dockless Shared Bikes: The Relationships among Incentive Forms, Intensity and Policy Compliance." Transportation, 2020, pp. 1-28.

26. Fukuda, Daisuke, and Shigeru Morichi. "Incorporating Aggregate Behavior in an Individual's Discrete Choice: An Application to Analyzing Illegal Bicycle Parking Behavior.” Transportation Research Part A-Policy and Practice, vol. 41, no. 4, 2007, pp. 313-325.

27. Han, Sun Sheng. "The Spatial Spread of Dockless Bike-Sharing Programs among Chinese Cities." Journal of Transport Geography, vol. 86, 2020, p. 102782 .

28. Peters, Luke, and Don MacKenzie. "The Death and Rebirth of Bikesharing in Seattle: Implications for Policy and System Design." Transportation Research
Part A-Policy and Practice, vol. 130, 2019, pp. 208226.

29. Vogel, Patrick, et al. "Understanding Bike-Sharing Systems Using Data Mining: Exploring Activity Patterns." Procedia - Social and Behavioral Sciences, vol. 20, 2011, pp. 514-523.

30. Ricci, Miriam. "Bike Sharing: A Review of Evidence on Impacts and Processes of Implementation and Operation." Research in Transportation Business and Management, vol. 15, 2015, pp. 28-38.

31. Moro, Suzana R., et al. "Barriers to Bicycle Sharing Systems Implementation: Analysis of Two Unsuccessful PSS.” Procedia CIRP, vol. 73, 2018, pp. 191-196.

32. Jiang, Qianling, et al. "Service Design Consideration of Solving the Problem of Disorderly-Parked Unbundled Sharing Bicycles with Low-Carbon Policy Orientation." 2018 IEEE International Conference on Applied System Invention (ICASI), 2018.

33. Chambers, Peter. "O Bike in Melbourne: A Plea for More Scepticism about Disruption and Capital, Based on What We Can Know about One Dockless Bike Scheme.” Transportation Research Part A-Policy and Practice, vol. 140, 2020, pp. 72-80.

34. Zhao, Daozhi, and Di Wang. "The Research of Tripartite Collaborative Governance on Disorderly Parking of Shared Bicycles Based on the Theory of Planned Behavior and Motivation Theories - A Case of Beijing, China." Sustainability, vol. 11, no. 19, 2019, p. 5431.

35. Niu Wenke. Multi-perspective analysis of the governance of bike-sharing [J]. Modern Management Science, 2020(02):86-88.

36. Yue Yujun, Hu Hanhui. Multi-theoretical analysis of urban bike-sharing governance [J]. Contemporary Economic Management, 2019,41(07):68-72.

37. Qin Zheng, Wang Qin. The tripartite coordination mechanism of sharing economic deduction: an example of bike-sharing [J]. Reform, 2017(05):124134.

38. Ma, Yuge, et al. "Challenges of Collaborative Governance in the Sharing Economy: The Case of Free-Floating Bike Sharing in Shanghai." Journal of Cleaner Production, vol. 197, 2018, pp. 356-365.

39. Guo Peng, Lin Xiangzhi, Huang Yi, Tu Siming, Bai Xiaoming, Yang Yawen, Ye Lin. bike-sharing: Collaborative Governance in Internet Technology and Public Services [J]. journal of public management, 2017,14(03):1-10+154.

40. [40] Li Qingyi, Long Shimin, Kang Yuee, Xu Wei. Study on continuous optimization of integrated management mode of urban bike-sharing-taking Chengdu as an example [J]. China Market, 2019(01):44-47. 